\title{
Syntax for Semantics: Krull's Maximal Ideal Theorem
}

\author{
Peter Schuster and Daniel Wessel
}

\begin{abstract}
Krull's Maximal Ideal Theorem (MIT) is one of the most prominent incarnations of the Axiom of Choice (AC) in ring theory. For many a consequence of AC, constructive counterparts are well within reach, provided attention is turned to the syntactical underpinning of the problem at hand. This is one of the viewpoints of the revised Hilbert Programme in commutative algebra, which will here be carried out for MIT and several related classical principles.
\end{abstract}

\section{Introduction}

Transfinite proof methods have been introduced into mathematics more than a century ago, most prominently by Ernst Zermelo's Well-Ordering Theorem (WO) dating back to 1904 (Zermelo 1904; 1908). To prove WO, Zermelo needed to postulate the Axiom of Choice (AC), about which Paul Lorenzen most appropriately remarked that by calling AC an axiom, "one believes oneself absolved from the responsibility to justify it." ${ }^{1}$ Though immediately causing a heated foundational debate, transfinite methods turned out soon after to work surprisingly well in mathematical practice, even for proving theorems of a finite nature. A typical proof method of the time was to use the principle of mathematical induction also beyond the

\footnotetext{
Peter Schuster

Dipartimento di Informatica, Università di Verona, Italy, e-mail: peter . schuster@univr . it

Daniel Wessel

Dipartimento di Informatica, Università di Verona, Italy, e-mail: daniel .wessel@univr.it

1 "Da man allerdings dort, wo angeblich keine Abzählung möglich ist, das Auswahlprinzip - indem man es als Axiom bezeichnet, glaubt man sich der Verpflichtung enthoben, es zu begründen - anwendet, lassen sich die Beweise für den ,abzählbaren' Fall meist ohne weiteres auf den ,überabzählbaren' Fall übertragen" (Lorenzen 1953, 241).
} 
context of natural numbers. This meant doing transfinite induction, and thus, more often than not, to invoke a well-ordering, the existence of which in general requires WO.

Soon after, Zermelo's postulate AC started to play a crucial role especially during the advent of abstract algebra, so already in 1910 for Steinitz's pure existence proof of the algebraic closure of an arbitrary field (Steinitz 1910). This methodical move raised considerable concern among the algebraists of the time, and Max Zorn allegedly spoke for many when he said in 1935:

The theorems of Steinitz concerning algebraic closure ... are barred, from the algebraic point of view, by the well-ordering theorem and its theory. (Zorn 1935, 667)

As a way out he coined what is now known as "Zorn's Lemma" (ZL) (Zorn 1935; Campbell 1978), to have a variant of WO more palatable to algebraists. Since then, ZL has gradually replaced WO in proof practice, especially in algebra.

Even for Wolfgang Krull, WO had merely been a means to an end, rather than an end in itself. Krull wrote to Heinrich Scholz on 18th April 1953: ${ }^{2}$

In working with the uncountable, in particular with the well-ordering theorem, I always had the feeling that one uses fictions there that need to be replaced some day by more reasonable concepts. But I was not getting upset over it, because I was convinced that in a careful application of the common "fictions", nothing false comes out, and because I was firmly counting on the man who would some day put all in order. Lorenzen has now found according to my conviction the right way ....

It is not unlikely that Krull had heard of Vaihinger's useful fictions (Vaihinger 1922; 1924), which gain justification already by practical usefulness rather than objective provability. Krull's lines may have been well received by Scholz, who was thoroughly versed in Vaihinger's philosophy of "As If" (Scholz 1919). According to Fine (1993, p. 3),

Vaihinger was trying to associate himself with an empiricist approach to positive, scientific knowledge and to disassociate his view from rationalism or Platonism; indeed from any view that would presume some reality to correspond to whatever the mind logically constructs.

The many successes of the revised Hilbert Programme in abstract algebra (Crosilla and Schuster 2014), as called for by Coquand and Lombardi (2006), seem to resonate well with Vaihinger's views. In fact, constructive counterparts of many a consequence of AC are within reach, provided we turn our attention to the syntactical underpinning of the respective problem at hand. It seems as if, for every fiction arising from transfinite methods, each concrete use in proof practice can be reduced to a use of the fiction's provable part.

\footnotetext{
2 Scholz-Archiv, Universitäts- und Landesbibliothek Münster, https : //www . uni-muenster .de/IVV5WS/ScholzWiki/doku.php?id=scans:blogs:ko-05-0647, accessed Nov. 15, 2018. Translation by Stefan Neuwirth (Coquand, Lombardi, and Neuwirth 2019).
} 
Our paper is meant to illustrate this with regard to Krull's (1929) Maximal Ideal Theorem (in the unital commutative case), presumably the most

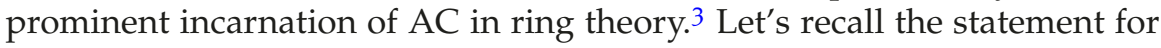
the sake of reference:

MIT Every non-trivial commutative ring with unit has a maximal ideal.

Incidentally, in today's textbooks, MIT is used to prove Steinitz's existence theorem, e.g., Kunz 1991.

More precisely, we develop an account of MIT in terms of an infinitary variant of Lorenzen's (1950, 1951, 1952, 1953) and Scott's (1971, 1973, 1974) entailment relations. No claim is made as to our treatment being the definitive one, nor do we intend to compete by any means with the sophisticated machinery of Dynamical Algebra (Coste, Lombardi, and Roy 2001; Lombardi and Quitté 2015; Yengui 2015), which, as we hasten to add, has already shown how to make constructive the use of maximal ideals (Yengui 2008; 2015).

We proceed as follows. In Section 2, we briefly discuss MIT in the case of countable, of Noetherian, and of arbitrary rings. In Section 3, we sketch a possible development of infinitary entailment relations (Wessel 2018), which will be applied in Section 4 to interpret MIT constructively. Several applications will be given in Section 5, which further illustrates that our generalised concept of entailment relation retains much of the conventional one's flexibility.

\section{On method and foundations}

Unless specifically stated otherwise, we work in Bishop's constructive mathematics, which can be formalised in Aczel's constructive set theory CZF (Aczel 1978; 1982; 1986; Aczel and Rathjen 2000; 2010). Recall that a set $S$ is said to be discrete if

$$
(\forall a, b \in S)(a=b \vee a \neq b) .
$$

A subset $T$ of $S$ is detachable if

$$
(\forall a \in S)(a \in T \vee a \notin T)
$$

By a finite set we understand a set that can be written as $\left\{a_{1}, \ldots, a_{n}\right\}$ for some $n \geq 0.4$ The set of all finite subsets of a set $S$ will be denoted by $\operatorname{Fin}(S)$,

\footnotetext{
3 Recall that MIT is in fact equivalent to AC over Zermelo-Fraenkel set theory ZF (Hodges 1979; Banaschewski 1994; Howard and Rubin 1998). In comparison, the Prime Ideal Theorem, asserting that every non-trivial commutative ring with unit has a prime ideal, is ZF-equivalent to the Boolean Ultrafilter Theorem (BUT) (Scott 1954; Banaschewski 1983), a weak form of AC (Howard and Rubin 1998).

4 For the sake of a slicker wording we thus deviate from the prevalent terminology of constructive mathematics and set theory (Aczel and Rathjen 2000; 2010; Bishop 1967;
} 
and the class of all subsets of $S$ by $\operatorname{Pow}(S)$. We further refer to Rinaldi, Schuster, and Wessel 2018 for provisos to carry over to the present paper.

We need to recall only few and basic notions from commutative ring theory (Lombardi and Quitté 2015; Yengui 2015). Throughout, let A denote a commutative ring with unit. If $U$ is a subset of $\mathbf{A}$, then $\langle U\rangle$ consists of all finite sums $r_{1} a_{1}+\cdots+r_{k} a_{k}$, where $r_{i} \in \mathbf{A}$ and $a_{i} \in U$. A subset $\mathfrak{a}$ of $\mathbf{A}$ is an ideal if $\langle\mathfrak{a}\rangle=\mathfrak{a}$. We say that elements $a_{1}, \ldots, a_{k} \in \mathbf{A}$ are comaximal if $1 \in\left\langle a_{1}, \ldots, a_{k}\right\rangle$. An ideal $\mathfrak{a}$ is proper if $1 \notin \mathfrak{a}$, and prime if it is proper and such that $a b \in \mathfrak{a}$ implies $a \in \mathfrak{a}$ or $b \in \mathfrak{a}$. The radical of an ideal $\mathfrak{a}$ is

$$
\sqrt{\mathfrak{a}}=\left\{a \in \mathbf{A} \mid(\exists n \in \mathbb{N})\left(a^{n} \in \mathfrak{a}\right)\right\} .
$$

An ideal $\mathfrak{a}$ is a radical ideal if $\sqrt{\mathfrak{a}}=\mathfrak{a}$. The Jacobson radical of an ideal $\mathfrak{a}$ is

$$
\operatorname{Jac}(\mathfrak{a})=\{a \in \mathbf{A} \mid(\forall b \in \mathbf{A})(\exists c \in \mathbf{A})(1-(1-a b) c \in \mathfrak{a})\} .
$$

In other words, $\operatorname{Jac}(\mathfrak{a})$ consists of all elements $a \in \mathbf{A}$ such that, for every $b \in \mathbf{A}$, the element $1-a b$ is invertible modulo $\mathfrak{a}$. Note that $\sqrt{\mathfrak{a}} \subseteq \operatorname{Jac}(\mathfrak{a})$. We write Jac $\mathbf{A}$ for $\operatorname{Jac}(0)$. Assuming $A C$, this Jac $\mathbf{A}$ is the intersection of all maximal ideals of A (Lombardi and Quitté 2015).

\section{Maximal ideals}

A commutative ring $\mathbf{A}$ is said to be a discrete field (Lombardi and Quitté 2015) if, for all $a \in \mathbf{A}$,

$$
a=0 \text { or } \quad(\exists b \in \mathbf{A})(a b=1) .
$$

For instance, the trivial ring in which $0=1$ is a discrete field, and nontriviality renders the carrier of $\mathbf{A}$ a discrete set. Now, let us define an ideal $\mathfrak{m}$ of $\mathbf{A}$ to be maximal if the quotient ring $\mathbf{A} / \mathfrak{m}$ is a non-trivial discrete field. This is to say that the maximal ideals of $\mathbf{A}$ are precisely the proper ideals $\mathfrak{m}$ of $\mathbf{A}$ which are complete, i.e., such that, for all $a \in \mathbf{A}$,

$$
a \in \mathfrak{m} \quad \text { or } \quad 1 \in \mathfrak{m}+\mathbf{A} a .
$$

Note that properness combined with completeness (1) ensures detachability.

Bishop and Bridges 1985; Mines, Richman, and Ruitenburg 1988; Lombardi and Quitté 2015): (1) to call 'subfinite' or 'finitely enumerable' a finite set in the sense above, i.e., a set $T$ for which there is a surjection from $\{1, \ldots, n\}$ to $T$ for some $n \geq 0$; and (2) to reserve the term 'finite' to sets which are in bijection with $\{1, \ldots, n\}$ for a necessarily unique $n \geq 0$. Also, finite sets in this stricter sense do not play a role in this paper. 


\section{The countable case}

From a constructive point of view, it is interesting to note that countable non-trivial commutative rings $\mathbf{A}$ do have maximal ideals whenever they are strongly discrete, i.e., whenever the finitely generated ideals are detachable (Mines, Richman, and Ruitenburg 1988). This is a standard argument, perhaps worth recalling: Let $a_{0}, a_{1}, \ldots$ be an enumeration of $\mathbf{A}$; inductively define a sequence $\left(\mathfrak{a}_{i}\right)_{i \in \mathbb{N}}$ of finitely generated proper ideals where $\mathfrak{a}_{0}=0$ and

put

$$
\mathfrak{a}_{i+1}= \begin{cases}\mathfrak{a}_{i} & \text { if } 1 \in \mathfrak{a}_{i}+\mathbf{A} a_{i} \\ \mathfrak{a}_{i}+\mathbf{A} a_{i} & \text { otherwise }\end{cases}
$$

$$
\mathfrak{m}=\bigcup_{i \in \mathbb{N}} \mathfrak{a}_{i} .
$$

This $\mathfrak{m}$ is a proper ideal, and since $a_{i} \in \mathfrak{m}$ if and only if $a_{i} \in \mathfrak{a}_{i+1}$, it follows that $\mathfrak{m}$ is maximal. ${ }^{5}$ Note that $\mathfrak{a}_{0}$ above can be replaced by any finitely generated proper ideal which needs to be extended to a maximal one.

Where generalisation demands that countability assumptions be dropped, $\mathrm{AC}$, be it in the guise of WO or ZL, comes to one's aid. Below, we show how to argue for MIT using Raoult's principle of Open Induction. It seems instructive to consider the case of Noetherian rings first.

\section{Noetherian rings}

Several constructive notions of Noetherianity have been proposed in the literature (Richman 1974; Seidenberg 1974; Mines, Richman, and Ruitenburg 1988; Jacobsson and Löfwall 1991; Coquand and Persson 1998a; Perdry 2004; Perdry and Schuster 2011; 2014; Blechschmidt 2017), the litmus test usually being Hilbert's Basis Theorem. Martin-Löf has proposed a definition replacing the Ascending Chain Condition by an induction principle (Jacobsson and Löfwall 1991), to be recalled in the following.

Let $(E, \leqslant)$ be a partially ordered set. A subset $H$ of $E$ is hereditary if

$$
(\forall x \in E)((\forall y \in E)(y<x \rightarrow y \in H) \rightarrow x \in H),
$$

where $y<x$ is understood as the conjunction of $y \leqslant x$ and $y \neq x$. The poset $E$ is well founded if its only hereditary subset is $E$ itself. A coherent ${ }^{6}$

\footnotetext{
5 We have adopted this from (Mines, Richman, and Ruitenburg 1988, Lemma vi.3.2). A similar argument is employed in classical reverse mathematics, as part of the proof that MIT for countable commutative rings is equivalent to $\mathrm{ACA}_{0}$ over $\mathrm{RCA}_{0}$ (Simpson 2009, III.5.).

${ }^{6} \mathbf{A}$ is coherent if for all $a_{1}, \ldots, a_{n} \in \mathbf{A}$, the kernel of the mapping $\left(x_{1}, \ldots, x_{n}\right) \mapsto a_{1} x_{1}+$ $\cdots+a_{n} x_{n}$ is finitely generated.
} 
ring in which every finitely generated ideal is detachable is said to be $M L$ Noetherian if the collection $\mathcal{I}_{\mathbf{A}}$ of finitely generated ideals, ordered by reverse set inclusion $\supseteq$, is well founded.

Suppose now that $\mathbf{A}$ has detachable ideals and $\left(\mathcal{I}_{\mathbf{A}}, \supseteq\right)$ is well founded. In analogy with primality tests (Perdry 2004), we further assume that there is a procedure which decides, given a finitely generated ideal $\mathfrak{a}$, whether it is maximal, and if not, gives an $a \in \mathbf{A}$ such that

$$
a \notin \mathfrak{a} \text { and } 1 \notin \mathfrak{a}+\mathbf{A} a .
$$

Consider the property $P(\mathfrak{a})$ that if $\mathfrak{a}$ is proper, then $\mathfrak{a}$ is contained in a maximal ideal. To show that $P$ applies to every $\mathfrak{a} \in \mathcal{I}_{\mathbf{A}}$, we need to show that it defines a hereditary subset of $\mathcal{I}_{\mathbf{A}}$. Thus, let $\mathfrak{a} \in \mathcal{I}_{\mathbf{A}}$ and suppose that $P(\mathfrak{b})$ for every $\mathfrak{b} \supsetneq \mathfrak{a}$. Now, either $\mathfrak{a}$ is maximal itself, so $P(\mathfrak{a})$ anyway. Else there is $a \notin \mathfrak{a}$ such that $\mathfrak{b}=\mathfrak{a}+\mathbf{A} a$ is proper by (3), whence $P(\mathfrak{b})$ by the assumption, and so again $P(\mathfrak{a})$.

It is worth noting that there is no need to invoke Dependent Choice, which would have been required had we assumed the classical Ascending Chain Condition instead of the induction principle.

\section{Proof by Open Induction}

The argument in the Noetherian case carries over almost literally to the general one, provided we work with Raoult's principle of Open Induction (OI) (Raoult 1988; Coquand 1997; Berger 2004; Schuster 2012; 2013; Rinaldi and Schuster 2016). Let us switch to ZFC.

Let $(E, \leqslant)$ be a partially ordered set with directed joins (a dcpo). Recall that a subset $O$ of $E$ is open (in the Scott topology; Johnstone 1982) if it is inaccessible by directed joins, which is to say that, if $D \subseteq E$ is directed and $\sup D \in O$, then $D \curlyvee O .7^{7}$ Now OI asserts that if $O \subseteq E$ is open and hereditary with respect to the converse $\geq$, then $O=E$. This principle follows classically from ZL, to which it is in fact equivalent by complementation (Raoult 1988; Rinaldi and Schuster 2016).

To see how OI applies to prove MIT, consider the dcpo of ideals of $\mathbf{A}$, where directed joins are unions. The property $P$ introduced in the preceding section describes, as we have seen, a hereditary subset, which is readily shown open. Therefore, by OI, every proper ideal is contained in a maximal one.

In order to interpret MIT constructively, we next turn it "upside down". In this guise it will assert the non-triviality of a suitable entailment relation, whose ideal elements are exactly the maximal ideals sought after. Abstract

7 The very intuitive notation $U \chi V$ has been borrowed from formal topology (Sambin 2003); it is to express that the sets $U$ and $V$ have an element in common. 
existence will thus be replaced by syntactic consistency, as called for in the revised Hilbert Programme.

\section{Entailment relations}

Entailment relations provide an astonishing example for the persistence of a mathematical concept which emerges independently in various fields of mathematical inquiry. Prevalently attributed to Scott (1971; 1973; 1974), similar concepts had been developed by Lorenzen (1950; 1951; 1952; 1953; 2017), as currently being revived by Coquand, Lombardi, and Neuwirth (2019; Coquand and Neuwirth 2017).

Let $S$ be a set. A relation

$$
\vdash \subseteq \operatorname{Fin}(S) \times \operatorname{Fin}(S)
$$

between finite subsets of $S$ is an entailment relation if it is reflexive, monotone, and transitive, as follows:

$$
\frac{U \ell^{\gamma} V}{U \vdash V}(\mathrm{R}) \quad \frac{U \vdash V}{U, U^{\prime} \vdash V, V^{\prime}}(\mathrm{M}) \quad \frac{U \vdash V, a \quad U, a \vdash V}{U \vdash V}
$$

where the usual shorthand notation is at work, putting a comma to abbreviate set union, and suppressing brackets where it should read a singleton set. We will refer to relations of this kind as "conventional" entailment relations, as opposed to the ones introduced below.

Notwithstanding the utmost versatility (Cederquist and Coquand 2000) of (conventional) entailment relations, some potential applications call for an extended concept which allows for arbitrary sets of succedents (rather than the usual finite ones). We sketch a possible development (Wessel 2018), rather close to the one of Fourman and Grayson 1982.

\section{An infinitary generalisation}

Let $S$ be a set. By a geometric entailment relation on $S$ we propose to understand a class relation

$$
\vdash \subseteq \operatorname{Fin}(S) \times \operatorname{Pow}(S)
$$

between finite and arbitrary subsets of $S$. This relation is required to be reflexive, monotone, and transitive in the following sense, respectively:

$$
\frac{U_{\ell} V^{\prime}}{U \vdash V}(\mathrm{R}) \quad \frac{U \vdash V}{U, U^{\prime} \vdash V, V^{\prime}}(\mathrm{M}) \quad \frac{U \vdash V \quad(\forall b \in V)(U, b \vdash W)}{U \vdash W}
$$


If $U \in \operatorname{Fin}(S)$ is such that $U \vdash$, i.e., $U \vdash \varnothing$, then $U$ is said to be inconsistent. Borrowing terminology from Dynamical Algebra (Coste, Lombardi, and Roy 2001), we say that $\vdash$ collapses if the empty set is inconsistent.

The recommended reading of $U \vdash V$ still is the same as for conventional entailment relations (Scott 1974), i.e., as that of a Gentzen sequent, or rather as

$$
\bigwedge_{a \in U} P(a) \rightarrow \bigvee_{b \in V} P(b),
$$

where $P$ is a distinguished predicate on $S$. However, the succedent $V$ must now be read as an infinite disjunction, so what we intend to capture are geometric sequents (Johnstone 2002), whence our choice of terminology. Henceforth, by "entailment relation" will always be meant "geometric entailment relation".

By an ideal element of $\vdash$ we understand a subset $\alpha$ of $S$ which is such that, if $U \vdash V$ and $U \subseteq \alpha$, then $\alpha \gamma V$. The class of all ideal elements of $\vdash$, its spectrum, will be denoted

$$
\operatorname{Spec}(\vdash) \text {. }
$$

Interestingly, Hertz (1922; 1923; 1929), presumably the first to conceive "the very idea of sequent calculi and of structural rules" (Legris 2012, 3), introduced the notion of ideal element with explicit reference to Vaihinger. Hertz's ideal elements refer to an "introduction of special symbols ... in order to reduce the complexity of axioms" (Legris 2012), whence there is a conceptual difference. The ideal elements in Hertz's (1922, §3.53. [Definition]) sense rather correspond to the elements of the generated frame (see below).

\section{Inductive generation and fundamental theorem}

Once a set $\mathcal{E}$ of initial entailments has been specified, how do we obtain the least entailment relation $\vdash_{\mathcal{E}}$ to contain $\mathcal{E}$ ? Impredicatively, $\vdash_{\mathcal{E}}$ would squarely be identified with the intersection of all entailment relations that contain $\mathcal{E}$. In order to not incur impredicativity, we are well advised to resort to an inductive definition instead, which constructive set theory conveniently accommodates (Aczel 1986; Aczel and Rathjen 2000; 2010; Rathjen 2005). To this end, a combination of strategies employed in formal topology (Coquand, Sambin, Smith, and Valentini 2003), with related ideas developed in sequent calculus (Negri 2014; Negri and von Plato 1998), has already proved viable (Wessel 2018).

The basic idea carries over from the recent treatment of conventional entailment relations (Rinaldi and Wessel 2019). To each "initial entailment" of $\mathcal{E}$ we assign an inference step, as follows: 


$$
(A, B) \in \mathcal{E} \quad \rightsquigarrow \quad \frac{\{(U \cup\{b\}, V) \mid b \in B\}}{(U \cup A, V)} \in \Phi
$$

Furthermore,

$$
\frac{\varnothing}{(U, V)} \in \Phi \quad \text { whenever } U_{\ell} \gamma .
$$

By the Class Inductive Definition Theorem (Aczel and Rathjen 2000), there is a smallest $\Phi$-closed class $I(\Phi)$, and we put $\vdash_{\mathcal{E}}=I(\Phi)$. This $\Phi$ is indeed an entailment relation; in fact, it is the least one to contain $\mathcal{E}$ (Wessel 2018).

Moreover, analogously to the finitary case (Cederquist and Coquand 2000), with every inductively generated entailment relation $\vdash_{\mathcal{E}}$, we can associate a certain complete distributive lattice $F$ together with a mapping $[\cdot]: S \rightarrow F$ such that, for all $U \in \operatorname{Fin}(S)$ and $V \in \operatorname{Pow}(S)$,

$$
U \vdash_{\mathcal{E}} V \text { if and only if } \bigwedge_{a \in U}[a] \leqslant \bigvee_{b \in V}[b],
$$

and which, moreover, has the evident universal property analogous to the fundamental result in Cederquist and Coquand 2000. ${ }^{8}$ Last but not least, the (set-generated) completely prime filters (Aczel 2006) of $F$ bijectively correspond to the ideal elements of $\vdash_{\mathcal{E}}$.

We can study this frame - which is a point-free presentation of the space of ideal elements equipped with the finite information topology (Fourman and Grayson 1982) - through its syntactic underpinning, i.e., by way of the entailment relation at hand.

Remark 1 If $\vdash_{\mathcal{E}}$ is generated by a set $\mathcal{E}$ of finitary axioms $(A, B)$, i.e., such that both $A, B \in \operatorname{Fin}(S)$, then, by an inductive argument,

$$
U \vdash_{\mathcal{E}} V \quad \text { if and only if }\left(\exists V_{0} \in \operatorname{Fin}(V)\right)\left(U \vdash_{\mathcal{E}} V_{0}\right) .
$$

Assuming AC, 9 by way of Scott's (1974) completeness theorem, every such entailment relation $\vdash$ is determined by its spectrum, i.e., such that

$$
U \vdash V \quad \text { if and only if } \quad(\forall \alpha \in \operatorname{Spec}(\vdash))(U \subseteq \alpha \rightarrow \alpha \gamma V) .
$$

In particular,

$$
\vdash a \text { if and only if } a \in \bigcap \operatorname{Spec}(\vdash) ;
$$

and non-collapse of $\vdash$ amounts to $\vdash$ having an ideal element. This, however, does not carry over to geometric entailment relations in general, due to

\footnotetext{
${ }^{8}$ Note that in CZF a frame invariably means a class frame, on account of certain size issues (Aczel 2006; Curi 2010). The focus should thus be put on the notion of a set-generated frame, of which the frame generated by a set $\mathcal{E}$ of axioms is an example, provided we go beyond CZF, adopting the Regular Extension Axiom REA.

9 Actually, BUT suffices, which is ZF-equivalent to Scott's completeness theorem (Rinaldi, Schuster, and Wessel 2018).
} 
the well-known fact from point-free topology that there are non-trivial non-spatial locales (Johnstone 1982).

Remark 2 Let $S$ and $T$ be sets equipped with entailment relations $\vdash$ and $\vdash^{\prime}$, respectively. Every function $f: S \rightarrow T$ such that, for all $U \in \operatorname{Fin}(S)$ and $V \in \operatorname{Pow}(S)$,

$$
U \vdash V \text { implies } f(U) \vdash^{\prime} f(V),
$$

induces a mapping of ideal elements

$$
f^{*}: \operatorname{Spec}\left(\vdash^{\prime}\right) \rightarrow \operatorname{Spec}(\vdash), \quad \alpha \mapsto f^{-1}(\alpha) .
$$

Note that if $f$ denotes a subset inclusion $S \subseteq T$, then $f^{*}$ amounts to restriction, i.e., $f^{*}(\alpha)=\alpha \cap S$. If moreover $S=T$, then $\vdash \subseteq \vdash^{\prime}$ entails $\operatorname{Spec}\left(\vdash^{\prime}\right) \subseteq \operatorname{Spec}(\vdash)$. In this manner, several extension theorems can be captured in terms of entailment relations (Cederquist and Coquand 2000; Rinaldi and Wessel 2018).

Definition 1 Let $\vdash$ and $\vdash^{\prime}$ be entailment relations on $S$ such that $\vdash \subseteq \vdash^{\prime}$. We say that $\vdash^{\prime}$ is weakly conservative over $\vdash$ if every $U \in \operatorname{Fin}(S)$ is inconsistent with respect to $\vdash$ if and only if it is so with respect to $\vdash^{\prime}$, i.e.,

$$
U \vdash \quad \text { if and only if } U \vdash^{\prime} .
$$

\section{Krull's theorem without choice}

\section{Prime ideals}

We first study the entailment relation of prime ideal, inductively generated by the following axioms (Cederquist and Coquand 2000):

$$
\begin{gathered}
1 \vdash_{\mathrm{p}} \\
a \vdash_{\mathrm{p}} a b, \\
a, b \vdash_{\mathrm{p}} a+b, \\
a b \vdash_{\mathrm{p}} a, b, \\
\vdash_{\mathrm{p}} 0 .
\end{gathered}
$$

The ideal elements of $\vdash_{\mathrm{p}}$ are precisely the prime ideals of $\mathbf{A}$. This entailment relation has a well-known direct, non-inductive description (Cederquist and Coquand 2000), sometimes known as formal Nullstellensatz:

Proposition 1 The following are equivalent:

1. $a_{1}, \ldots, a_{k} \vdash_{\mathrm{p}} b_{1}, \ldots, b_{\ell}$. 
2. $b_{1} \cdots b_{\ell} \in \sqrt{\left\langle a_{1}, \ldots, a_{k}\right\rangle}$.

The formal Nullstellensatz has many applications in constructive algebra (Coquand 2009), e.g., to give an elementary constructive proof of the GaußJoyal Lemma (Banaschewski and Vermeulen 1996), the classical proof of which proceeds by reduction modulo a generic prime ideal (Eisenbud 1995).

Often the restriction of an entailment relation to its trace, i.e., to its singleconclusion instances, promotes further insight. The entailment relation of radical ideal is generated by the following axioms (Rinaldi, Schuster, and Wessel 2017; 2018):

$$
\begin{aligned}
a & \triangleright a b, \\
a, b & \triangleright a+b, \\
a^{2} & \triangleright a, \\
& \triangleright 0 .
\end{aligned}
$$

The ideal elements of $\triangleright$ are precisely the radical ideals of $\mathbf{A}$, and it is easy to see that

$$
a_{1}, \ldots, a_{k} \triangleright b \text { if and only if } a_{1}, \ldots, a_{k} \vdash_{\mathrm{p}} b .
$$

In other words, the (multi-conclusion) entailment relation of prime ideal is a conservative extension of the (single-conclusion) entailment relation of radical ideal (Rinaldi, Schuster, and Wessel 2017; 2018). This conservation boils down to applications of the well-known but seldom made explicit (Rinaldi 2014; Rinaldi and Schuster 2016) computational rule that

$$
\sqrt{\langle U, a\rangle} \cap \sqrt{\left\langle U^{\prime}, b\right\rangle} \subseteq \sqrt{\left\langle U, U^{\prime}, a b\right\rangle} .
$$

The semantic counterpart of this conservation result is that every radical ideal is the intersection of all prime ideals containing it, also known as Krull's Lemma. Again, classical arguments which proceed via the latter can sometimes be reshaped in a constructive manner using (7) (Wessel 2020).

\section{Maximal ideals}

Next we consider the entailment relation of maximal ideal of $\mathbf{A}$, generated by the following axioms:

$$
\begin{aligned}
& 1 \vdash_{\mathrm{m}} \\
& a \vdash_{\mathrm{m}} a b, \\
& a, b \vdash_{\mathrm{m}} a+b, \\
& \quad \vdash_{\mathrm{m}} a,\{1-a b \mid b \in \mathbf{A}\} .
\end{aligned}
$$


The ideal elements of $\vdash_{\mathrm{m}}$ are exactly the maximal ideals of $\mathbf{A}$ in the sense of Section 2. We have not postulated $\vdash_{\mathrm{m}} 0$, for it can be readily inferred by instantiation of the last axiom for $a=0$, which yields $\vdash_{\mathrm{m}} 0,1$ and which in turn can be cut with $1 \vdash_{\mathrm{m}}$.

Classically, in view of MIT, this entailment relation has an ideal element, due to which it does not collapse. Our next aim is to give an elementary constructive proof of the latter.

Let us first capture the classical fact that every maximal ideal is prime.

Lemma $\mathbf{1}$ For all $a, b \in \mathbf{A}$,

$$
a b \vdash_{\mathrm{m}} a, b .
$$

In particular, $\vdash_{\mathrm{p}} \subseteq \vdash_{\mathrm{m}}$.

Proof For all $c \in \mathbf{A}, a b c+(1-a c) b=b$, which witnesses $a b, 1-a c \vdash_{\mathrm{m}} b$. This can be used to cut $\vdash_{\mathrm{m}} a,\{1-a c \mid c \in A\}$ so as to obtain $a b \vdash_{\mathrm{m}} a, b$.

In view of Remark 2, Lemma 1 entails in fact that $\operatorname{Spec}\left(\vdash_{\mathrm{m}}\right) \subseteq \operatorname{Spec}\left(\vdash_{\mathrm{p}}\right)$. In other words, every maximal ideal is prime.

Lemma 2 For all $a \in \mathbf{A}, U \in \operatorname{Fin}(\mathbf{A})$, and $V \in \operatorname{Pow}(\mathbf{A})$, the following are equivalent:

1. $U \vdash_{\mathrm{m}} a, V$.

2. $(\forall b \in \mathbf{A})\left(U, 1-a b \vdash_{\mathrm{m}} V\right)$.

Proof Suppose that $U \vdash_{\mathrm{m}} a, V$ and let $b \in \mathbf{A}$. Since $1-a b, a \vdash_{\mathrm{m}}$ due to Proposition 1 and Lemma 1 , we get $U, 1-a b \vdash_{\mathrm{m}} V$ by cut. Conversely, if $U, 1-a b \vdash_{\mathrm{m}} V$ for all $b \in \mathbf{A}$, then $\vdash_{\mathrm{m}} a,\{1-a b \mid b \in \mathbf{A}\}$ can be cut so as to obtain $U \vdash_{\mathrm{m}} a, V$.

Lemma 3 The following are equivalent:

1. $a_{1}, \ldots, a_{k} \vdash_{\mathrm{m}}$.

2. $1 \in\left\langle a_{1}, \ldots, a_{k}\right\rangle$.

In particular, $a \vdash_{\mathrm{m}}$ if and only if $a$ is invertible.

Proof If $1 \in\left\langle a_{1}, \ldots, a_{k}\right\rangle$, then $a_{1}, \ldots, a_{k} \vdash_{\mathrm{p}}$ by Proposition 1 and thus $a_{1}, \ldots, a_{k} \vdash_{\mathrm{m}}$ by Lemma 1 . As regards the converse, it is enough to check that

$$
\frac{U \vdash_{\mathrm{m}} V \quad(\forall b \in V)\left(1 \in\left\langle a_{1}, \ldots, a_{k}, b\right\rangle\right)}{1 \in\left\langle a_{1}, \ldots, a_{k}, U\right\rangle}
$$

where for $U \vdash_{\mathrm{m}} V$ it suffices to instantiate initial entailments only (Wessel 2018). Except perhaps for the axiom of maximality, this is trivial. Hence, let $a \in \mathbf{A}$ and suppose that

$$
1 \in\left\langle a_{1}, \ldots, a_{k}, a\right\rangle
$$

as well as that, for all $b \in \mathbf{A}$, 


$$
1 \in\left\langle a_{1}, \ldots, a_{k}, 1-a b\right\rangle
$$

According to the former, there is $b \in \mathbf{A}$ such that $1-a b \in\left\langle a_{1}, \ldots, a_{k}\right\rangle$, which, if instantiated within the latter assumption, yields the desired conclusion $1 \in\left\langle a_{1}, \ldots, a_{k}\right\rangle$.

In view of Proposition 1 , Lemma 3 implies that a finite subset $U$ of $\mathbf{A}$ is inconsistent with respect to $\vdash_{\mathrm{m}}$ precisely if it is so with respect to $\vdash_{\mathrm{p}}$, i.e.,

$$
U \vdash_{\mathrm{p}} \quad \text { if and only if } \quad U \vdash_{\mathrm{m}} .
$$

For this we say that $\vdash_{\mathrm{m}}$ is a weakly conservative extension of $\vdash_{\mathrm{p}}$. In particular (Wessel 2018), the classical counterpart using AC is that every prime ideal is contained in a maximal ideal, a form of Krull's MIT.

Corollary $\mathbf{1}$ The following are equivalent:

1. $\vdash_{\mathrm{m}}$ collapses.

2. A is trivial.

Proof According to Lemma $3, \vdash_{\mathrm{m}}$ collapses if and only if $1 \in\langle 0\rangle$.

In all, non-collapse of $\vdash_{\mathrm{m}}$ is immediate, provided that $\mathbf{A}$ is a non-trivial ring to begin with, which is a constructive counterpart of MIT. We hasten to add that Coquand and Persson (1998b), too, have obtained a constructive interpretation for the existence of maximal ideals using constructive models based on point-free topology.

Last but not least, putting together the preceding lemmas, we arrive at a direct, non-inductive description of the canonical finitary subrelation of $\vdash_{\mathrm{m}}$, analogous to the formal Nullstellensatz (Proposition 1).

Proposition 2 The following are equivalent:

1. $a_{1}, \ldots, a_{k} \vdash_{\mathrm{m}} b_{1}, \ldots, b_{\ell}$.

2. $b_{1} \cdots b_{\ell} \in \operatorname{Jac}\left(\left\langle a_{1}, \ldots, a_{k}\right\rangle\right)$.

Proof Since $U \vdash_{\mathrm{m}} a, b$ if and only if $U \vdash_{\mathrm{m}} a b$, which is a consequence of Lemma 1 , it suffices to consider the single-conclusion case $\ell=1$. Combining the case $V=\varnothing$ of Lemma 2 with Lemma 3, we see that

$$
a_{1}, \ldots, a_{k} \vdash_{\mathrm{m}} b \quad \text { if and only if } \quad(\forall c \in \mathbf{A})\left(1 \in\left\langle a_{1}, \ldots, a_{k}, 1-b c\right\rangle\right),
$$

the latter of which is to say that $1-b c$ is invertible modulo $\left\langle a_{1}, \ldots, a_{k}\right\rangle$ for any $c \in \mathbf{A}$.

Corollary 2 The following are equivalent:

1. $\vdash_{\mathrm{m}} a$.

2. $a \in \mathrm{Jac} \mathbf{A}$. 
Corollary 2 is reminiscent of the classical fact that Jac $\mathbf{A}$ is the intersection of all maximal ideals of $\mathbf{A}$.

Remark 3 The condition on $\mathbf{A}$ to be a residually discrete local ring (Lombardi and Quitte 2015; Yengui 2015), i.e., that every $a \in \mathbf{A}$ either is invertible or belongs to the Jacobson radical Jac $\mathbf{A}$, can here be captured in terms of the completeness (Scott 1974) of $\vdash_{\mathrm{m}}$, which is to say that, for all $a \in \mathbf{A}$,

$$
a \vdash_{\mathrm{m}} \text { or } \vdash_{\mathrm{m}} a \text {. }
$$

Notice that because of $1-a b, a \vdash_{\mathrm{m}}$ for any $b \in \mathbf{A}$, if $\vdash_{\mathrm{m}}$ is complete, then $a \vdash_{\mathrm{m}}$ or $1-a b \vdash_{\mathrm{m}}$, which of course follows directly from the description of Jac $\mathbf{A}$ as well. In particular,

$$
a \vdash_{\mathrm{m}} \text { or } 1-a \vdash_{\mathrm{m}}
$$

which condition, in view of Lemma 3 above, translates back as asserting that A be a local ring (Lombardi and Quitté 2015; Yengui 2015).

\section{Applications}

\section{Krull dimension}

In classical mathematics, the Krull dimension of a ring or distributive lattice is defined as the supremum of the lengths of strictly increasing chains of prime ideals. As it takes recourse to objects which may fail to exist constructively, this definition is not effective. A constructive definition was suggested by Joyal (Boileau and Joyal 1981; Español 1982) ${ }^{10}$ and has been analysed also from the point of view of entailment relations (Cederquist and Coquand 2000). Subsequent developments (Lombardi 2002; Coquand and Lombardi 2002) have led to an inductive definition of dimension (Coquand, Lombardi, and Roy 2005), which via Stone duality applies just as well to spectral spaces, in which case it appears as a simplified version of the Menger-Urysohn definition (Coquand, Lombardi, and Roy 2005).

Here is the constructive and elementary characterisation of Krull dimension (Kdim) (Lombardi 2002; Coquand and Lombardi 2002; Coquand, Lombardi, and Roy 2005), by now widely used (Yengui 2015; Kemper and Yengui 2019): let $\ell \in \mathbb{N}$, then $K \operatorname{dim} \mathbf{A} \leqslant \ell$ if and only if, for all $a_{0}, \ldots, a_{\ell} \in \mathbf{A}$, there are $b_{0}, \ldots, b_{\ell} \in \mathbf{A}$ and $m_{0}, \ldots, m_{\ell} \in \mathbb{N}$ such that

$$
a_{0}^{m_{0}}\left(\cdots\left(a_{\ell}^{m_{\ell}}\left(1+a_{\ell} b_{\ell}\right)+\cdots\right)+a_{0} b_{0}\right)=0 .
$$

\footnotetext{
${ }^{10}$ With this, as Lombardi and Quitte put it, "the theory of dimension which seemed bathed in ethereal spaces - that are invisible when you do not trust the axiom of choice has become (at least in principle) an elementary theory, without any further mysteries" (Lombardi and Quitté 2015, p. xliii).
} 
In particular,

$\operatorname{Kdim} \mathbf{A} \leqslant 0 \quad$ if and only if $\quad(\forall a \in \mathbf{A})(\exists n \in \mathbb{N})(\exists b \in \mathbf{A})\left(a^{n}(1-a b)=0\right)$.

For example, if $\mathbf{A}$ is a Boolean ring, i.e., such that $a^{2}=a$ for every $a \in \mathbf{A}$, then evidently Kdim $\mathbf{A} \leqslant 0 .{ }^{11}$ The following Proposition 3 captures the classical counterpart of $\mathrm{Kdim} \mathbf{A} \leqslant 0$, which by Remark 2 asserts that every prime ideal of $\mathbf{A}$ is a maximal ideal, or in other words, that there is no proper chain $\mathfrak{p}_{0} \subsetneq \mathfrak{p}_{1}$ of prime ideals. For a similar characterisation, see Cederquist and Coquand 2000, Corollary 4.

Proposition 3 The following are equivalent:

1. $\operatorname{Kdim} \mathbf{A} \leqslant 0$.

2. $\vdash_{\mathrm{p}}=\vdash_{\mathrm{m}}$.

Proof Suppose that Kdim A $\leqslant 0$. Due to Lemma 1, in order to show $\vdash_{\mathrm{p}}=\vdash_{\mathrm{m}}$, it suffices to prove $\vdash_{\mathrm{p}} \supseteq \vdash_{\mathrm{m}}$, i.e., that, for every $a \in \mathbf{A}$,

$$
\vdash_{\mathrm{p}} a,\{1-a b \mid b \in \mathbf{A}\} .
$$

Thus, let $a \in \mathbf{A}$. Since $\operatorname{Kdim} \mathbf{A} \leqslant 0$, there is $n \in \mathbb{N}$ and $b \in \mathbf{A}$ such that $a^{n}(1-a b)=0$, whence $\vdash_{\mathrm{p}} a, 1-a b$, and monotonicity $(\mathrm{M})$ yields the desired entailment. Conversely, if indeed $\vdash_{\mathrm{p}}=\vdash_{\mathrm{m}}$, then, since $\vdash_{\mathrm{p}}$ is finitary (cf. Remark 1), for every $a \in \mathbf{A}$ there are $b_{1}, \ldots, b_{\ell} \in \mathbf{A}$ such that

$$
\vdash_{\mathrm{p}} a, 1-a b_{1}, \ldots, 1-a b_{\ell} \text {. }
$$

Hence, according to Proposition 1,

$$
a\left(1-a b_{1}\right) \cdots\left(1-a b_{k}\right) \in \sqrt{0} .
$$

Since $\Pi_{j=1}^{\ell}\left(1-a b_{j}\right)^{n}=1-a c$ for a suitable $c \in A$, this completes the proof. $\square$

\section{Jacobson rings}

Rings with Krull dimension $\leqslant 0$ are among those for which $\vdash_{\mathrm{m}}$ is conservative over the single-conclusion entailment relation $\triangleright$ of radical ideal (cf. Section 4) in the sense that

$$
U \vdash_{\mathrm{m}} a \text { if and only if } U \triangleright a .
$$

\footnotetext{
${ }^{11}$ Incidentally, as Moore points out with regard to Stone's development of duality theory, "it was apropos of Boolean rings, which Krull did not consider specifically, that [MIT] proved to be particularly significant" (Moore 1982, p. 229).
} 
This is immediate in view of Propositions 3 and 1 . However, as opposed to the case of primality (Rinaldi, Schuster, and Wessel 2017; 2018), conservation of maximality (8) does not hold in general.

In this context it might be interesting to consider Jacobson rings, ${ }^{12}$ a classical characterisation of which is that every prime ideal be the intersection of all maximal ideals containing it. This can be rephrased as demanding that the two notions of radical coincide (Goldman 1951), which is to say that, for every ideal $\mathfrak{a}$ of $\mathbf{A}$,

$$
\operatorname{Jac}(\mathfrak{a})=\sqrt{\mathfrak{a}} .
$$

The latter characterisation can be recast in terms of entailment relations. This is really just a back and forth between entailment and the corresponding algebraic certificate, but perhaps worth carrying out for the sake of illustration.

Proposition 4 The following are equivalent:

1. A is a Jacobson ring.

2. $\vdash_{\mathrm{m}}$ is conservative over $\triangleright$ and the Jacobson radical is a finitary closure operator.

Proof If the Jacobson radical coincides with the nilradical, then of course the former is finitary (as so is the latter), and $\vdash_{\mathrm{m}}$ is conservative over $\triangleright$ on account of (6) and Propositions 1 and 2. Conversely, if conservation holds and the Jacobson radical is a finitary closure operator, then for each ideal $\mathfrak{a}$ and every $a \in \operatorname{Jac}(\mathfrak{a})$ there are $a_{1}, \ldots, a_{k} \in \mathfrak{a}$ such that $a \in \operatorname{Jac}\left(\left\langle a_{1}, \ldots, a_{k}\right\rangle\right)$, so $a_{1}, \ldots, a_{k} \vdash_{\mathrm{m}} a$, hence $a_{1}, \ldots, a_{k} \triangleright a$, which implies $a \in \sqrt{\mathfrak{a}}$.

\section{Primary ideals and von Neumann regularity}

Recall that a (not necessarily commutative) ring $\mathbf{A}$ is said to be von Neumann regular if

$$
(\forall a \in \mathbf{A})(\exists b \in \mathbf{A})(a=a b a) .
$$

Every commutative von Neumann regular ring $\mathbf{A}$ is reduced, i.e.,

$$
(\forall a \in \mathbf{A})\left(a^{2}=0 \rightarrow a=0\right) .
$$

In fact, $\mathbf{A}$ is von Neumann regular if and only if $\mathbf{A}$ is reduced and $K \operatorname{dim} \mathbf{A} \leqslant 0$. In a commutative von Neumann regular ring $\mathbf{A}$ every primary ideal is

\footnotetext{
12 "This name, bestowed by Krull, honors Nathan Jacobson's studies of the intersection of the maximal ideals of a ring, which is now called the Jacobson radical" (Eisenbud 1995, 132, fn. 1). Goldman (1951), independently and in view of the general form of Hilbert's Nullstellensatz, had chosen the name Hilbert ring.
} 
maximal (Satyanarayana 1967; Lal 1971). This lends itself to be captured in terms of entailment relations. ${ }^{13}$

We consider the entailment relation of primary ideal, generated by

$$
\begin{aligned}
& 1 \vdash_{\mathrm{p}^{\prime}}, \\
& a \vdash \mathrm{p}^{\prime} a b, \\
& a, b \vdash \mathrm{p}^{\prime} a+b, \\
& a b \vdash_{\mathrm{p}^{\prime}} a,\left\{b^{n} \mid n>0\right\}, \\
& \vdash_{\mathrm{p}^{\prime}} 0 .
\end{aligned}
$$

The ideal elements of $\vdash_{p^{\prime}}$ are exactly the primary ideals of $\mathbf{A}$, i.e., the proper ideals $\mathfrak{a}$ of $\mathbf{A}$ such that if $a b \in \mathfrak{a}$, then $a \in \mathfrak{a}$ or $b^{n} \in \mathfrak{a}$ for some $n>0$. that

Since evidently $a b \vdash_{\mathrm{p}} a,\left\{b^{n} \mid n>0\right\}$, and in view of Lemma 1 , it is clear

$$
\vdash_{\mathrm{p}^{\prime}} \subseteq \vdash_{\mathrm{p}} \subseteq \vdash_{\mathrm{m}}
$$

Moreover, following a strategy similar to the one employed for the proof of Lemma 3, it can be shown that

$$
a_{1}, \ldots, a_{k} \vdash_{\mathrm{p}^{\prime}} \quad \text { if and only if } \quad 1 \in\left\langle a_{1}, \ldots, a_{k}\right\rangle,
$$

whence both $\vdash_{\mathrm{p}}$ and $\vdash_{\mathrm{m}}$ are weakly conservative over $\vdash_{\mathrm{p}^{\prime}}$.

Similar to the case of nil- and Jacobson radicals, the Gilmer radical of $\mathbf{A}$ (Brewer and Richman 2006), which is the intersection of all primary ideals of A, could be described by means of the "tautologies" for the corresponding entailment relation, i.e., as the set of all $a \in \mathbf{A}$ such that $\vdash_{\mathrm{p}^{\prime}} a$. Classically, it is known that the Gilmer radical of $\mathbf{A}$ is zero if and only if $\mathbf{A}$ is a subring of a product of zero-dimensional rings (Arapović 1983; Brewer and Richman 2006).

The following is straightforward and presented just for the sake of practice. Its converse will briefly be discussed below in Remark 4, but from a classical perspective.

Proposition 5 If $\mathbf{A}$ is von Neumann regular, then $\vdash_{\mathrm{p}^{\prime}}=\vdash_{\mathrm{m}}$.

Proof It suffices to check $\vdash_{\mathrm{p}^{\prime}} \supseteq \vdash_{\mathrm{m}}$, i.e., that, for all $a \in \mathbf{A}$,

$$
\vdash_{\mathrm{p}^{\prime}} a,\{1-a b \mid b \in \mathbf{A}\} \text {. }
$$

Thus, let $a \in \mathbf{A}$. By von Neumann regularity, there is $b \in \mathbf{A}$ such that $a(1-a b)=0$. It follows that

$$
\vdash_{\mathrm{p}^{\prime}} a,\left\{(1-a b)^{n} \mid n>0\right\} .
$$

${ }^{13}$ Rings in which every primary ideal is maximal have been studied under the name P-ring (Satyanarayana 1967; Lal 1971). 
Since $(1-a b)^{n}=1-a c$ for a suitable $c \in \mathbf{A}$, the latter entailment yields the desired one by monotonicity $(\mathrm{M})$.

Remark 4 That every primary ideal of A be a maximal ideal is not only necessary for von Neumann regularity, but actually sufficient (Lal 1971). Unfortunately, we don't have at hand an elementary argument solely in terms of entailment relations; but here is a brief and succinct classical one: if $\vdash_{\mathrm{m}}=\vdash_{\mathrm{p}^{\prime}}$, then, on the one hand, $\vdash_{\mathrm{m}}=\vdash_{\mathrm{p}}$, whence $\operatorname{Kdim} \mathbf{A} \leqslant 0$ by Proposition 3. On the other hand, $\vdash_{\mathrm{p}}=\vdash_{\mathrm{p}^{\prime}}$, which directly implies that A is reduced since, according to the above-mentioned result of Arapović 1983 and Brewer and Richman 2006.

\section{Integral extensions}

Recall that if $\phi: \mathbf{A} \rightarrow \mathbf{B}$ is a homomorphism of commutative rings, and if $\mathfrak{p}$ is a prime ideal of $\mathbf{B}$, then the pre-image $\phi^{-1}(\mathfrak{p})$ is a prime ideal of $\mathbf{A}$. In general, this does not carry over if "prime" is to be replaced with "maximal" (Atiyah and Macdonald 1969). However, it is a standard observation that maximal ideals do pull back along integral ring extensions. Let's capture this in terms of entailment relations.

Let $\mathbf{A} \subseteq \mathbf{A}^{\prime}$ be commutative rings. Recall that an element $x \in \mathbf{A}^{\prime}$ is said to be integral over $\mathbf{A}$ if

$$
x^{n}=a_{1} x^{n-1}+a_{2} x^{n-2}+\cdots+a_{n-1} x+a_{n}
$$

for certain $a_{1}, \ldots, a_{n} \in \mathbf{A}$ and $n>0$. The ring $\mathbf{A}^{\prime}$ is said to be integral over $\mathbf{A}$ if every element of $\mathbf{A}^{\prime}$ is integral over $\mathbf{A}$. In the following, we assume that $\mathbf{A}^{\prime}$ is integral over $\mathbf{A}$.

We consider the entailment relations $\vdash_{\mathrm{m}}$ and $\vdash_{\mathrm{m}}^{\prime}$ of maximal ideals of A and $\mathbf{A}^{\prime}$, respectively. The question which poses itself is whether $\vdash_{\mathrm{m}} \subseteq \vdash_{\mathrm{m}}^{\prime}$. For this to be answered affirmatively, we only need to check that the maximality axiom interprets, which is to say that, for all $a \in \mathbf{A}$,

$$
\vdash_{\mathrm{m}}^{\prime} a,\{1-a b \mid b \in \mathbf{A}\} \text {. }
$$

The following lemma is straightforward and actually holds for every entailment relation considered in this note; the one after is crucial in the above regard.

Lemma 4 Let $a, b, c \in \mathbf{A}$ and $n>0$.

1. $1-a \vdash_{\mathrm{m}} 1-a^{n}$.

2. $1-a, a b+c \vdash_{\mathrm{m}} b+c$.

Proof The first entailment is witnessed by $1-a^{n}=(1-a)\left(1+a+\cdots+a^{n-1}\right)$, and the second by $(1-a) b+a b+c=b+c$. 
Lemma 5 Let $\mathbf{A}^{\prime}$ be integral over $\mathbf{A}$, and let $a \in \mathbf{A}$. For every $x \in \mathbf{A}^{\prime}$ there is $b \in \mathbf{A}$ such that

$$
1-a x \vdash_{\mathrm{m}}^{\prime} 1-a b .
$$

Proof To begin with, write

$$
x^{n}=a_{1} x^{n-1}+a_{2} x^{n-2}+\cdots+a_{n-1} x+a_{n}
$$

for certain $n>0$ and $a_{i} \in \mathbf{A}$. Lemma 4.1 implies $1-a x \vdash_{\mathrm{m}}^{\prime} 1-(a x)^{n}$, which is

$$
1-a x \vdash_{\mathrm{m}}^{\prime} 1-a\left((a x)^{n-1} a_{1}+(a x)^{n-2} a a_{2}+\cdots+(a x) a^{n-2} a_{n-1}+a^{n-1} a_{n}\right) .
$$

By way of Lemma 4.2 and cut (T), we may now cancel the $a x^{\prime}$ s in the succedent, which yields

$$
1-a x \vdash_{\mathrm{m}}^{\prime} 1-a\left(a_{1}+a a_{2}+\cdots+a^{n-1} a_{n}\right),
$$

so that $b=a_{1}+a a_{2}+\cdots+a^{n-1} a_{n} \in \mathbf{A}$ witnesses the claim.

Proposition 6 Let $\mathbf{A}^{\prime}$ be integral over $\mathbf{A}$.

1. $\vdash_{\mathrm{m}} \subseteq \vdash_{\mathrm{m}}^{\prime}$.

2. $\vdash_{\mathrm{m}}^{\prime}$ is weakly conservative over $\vdash_{\mathrm{m}}$.

Proof 1. Let $a \in \mathbf{A}$. First we instantiate the maximality axiom with respect to $\mathbf{A}^{\prime}$, i.e.,

$$
\vdash_{\mathrm{m}}^{\prime} a,\left\{1-a x \mid x \in \mathbf{A}^{\prime}\right\} \text {. }
$$

According to Lemma 5 , for every $x \in \mathbf{A}^{\prime}$ there is $b \in \mathbf{A}$ such that $1-a x \vdash_{\mathrm{m}}^{\prime}$ $1-a b$, whence, by monotonicity $(\mathrm{M})$,

$$
1-a x \vdash_{\mathrm{m}}^{\prime}\{1-a b \mid b \in \mathbf{A}\} \text {. }
$$

Transitivity (T) yields

$$
\vdash_{\mathrm{m}}^{\prime} a,\{1-a b \mid b \in \mathbf{A}\} .
$$

2. In view of Lemma 3 , this is just another way of expressing that if elements $a_{1}, \ldots, a_{k} \in \mathbf{A}$ are comaximal in $\mathbf{A}^{\prime}$ then they are so with respect to $\mathbf{A}$. This can be shown by induction, passing for $k>0$ to the quotients

$$
\mathbf{A} /\left(\mathbf{A} \cap \mathbf{A}^{\prime} a_{k}\right) \hookrightarrow \mathbf{A}^{\prime} / \mathbf{A}^{\prime} a_{k}
$$

and taking into account that $\mathbf{A} \cap \mathbf{A}^{\prime} a_{k} \subseteq \sqrt{\left\langle a_{k}\right\rangle}$ (Coquand, Ducos, Lombardi, and Quitté 2009, Lemma 2.1).

How do we interpret Proposition 6? By Clause 1 and Remark 2, there is an induced mapping of ideal elements

$$
\operatorname{Spec}\left(\vdash_{\mathrm{m}}^{\prime}\right) \rightarrow \operatorname{Spec}\left(\vdash_{\mathrm{m}}\right), \quad \mathfrak{m} \mapsto \mathfrak{m} \cap \mathbf{A} .
$$


In other words, restriction preserves maximality. Assuming AC, by Clause 2, this mapping is surjective (Wessel 2018)!

\section{Acknowledgements}

The research that has led to this paper was carried out within the project "A New Dawn of Intuitionism: Mathematical and Philosophical Advances" (ID 60842) funded by the John Templeton Foundation. Partial support has come from the programme "Dipartimenti di Eccellenza 2018-2022" of the Italian Ministry of Education, Universities and Research (MIUR), and the project "Categorical localisation: methods and foundations" (CATLOC) funded by the Università degli Studi di Verona within the programme "Ricerca di Base 2015". The final version of this paper was prepared within the project "Reducing complexity in algebra, logic, combinatorics - REDCOM" belonging to the programme "Ricerca Scientifica di Eccellenza 2018" of the Fondazione Cariverona. ${ }^{14}$ Essential parts of this paper were conceived when both authors visited the Hausdorff Research Institute for Mathematics (HIM), University of Bonn, on the occasion of the trimester program "Types, Sets, and Constructions", May-August 2018. The related financial support is gratefully acknowledged.

The authors express their gratitude for interesting discussions and valuable hints to Ingo Blechschmidt, who was so kind as to have a look at the manuscript, as well as to Thierry Coquand, Henri Lombardi, Stefan Neuwirth, Davide Rinaldi, Ihsen Yengui, and, last but not least, to the referee for expertly remarks and an appreciative reading of the manuscript.

\section{References}

Aczel, Peter. 1978. "The type theoretic interpretation of constructive set theory." In Logic Colloquium '77 (Proc. Conf., Wroctaw, 1977), volume 96 of Studies in logic and the foundations of mathematics, pages 55-66. Amsterdam: North-Holland.

. 1982. "The type theoretic interpretation of constructive set theory: Choice principles." In The L. E. J. Brouwer Centenary Symposium (Noordwijkerhout, 1981), volume 110 of Studies in logic and the foundations of mathematics, pages 1-40. Amsterdam: North-Holland.

1986. "The type theoretic interpretation of constructive set theory: Inductive definitions." In Logic, methodology and philosophy of science, vII (Salzburg, 1983), volume 114 of Studies in logic and the foundations of mathematics, pages 17-49. Amsterdam: North-Holland.

14 The opinions expressed in this paper are those of the authors and do not necessarily reflect the views of those foundations. 
. 2006. "Aspects of general topology in constructive set theory." Annals of Pure and Applied Logic 137 (1-3): 3-29.

Aczel, Peter, and Michael Rathjen. 2000. "Notes on constructive set theory." Technical report, Institut Mittag-Leffler. Report No. 40.

- 2010. "Constructive set theory." Book draft. URL: https://www1 . maths. leeds.ac.uk/ rathjen/book. pdf.

Arapović, Miroslav. 1983. "On the embedding of a commutative ring into a 0-dimensional commutative ring." Glasnik Matematički Ser. III 18 (1): 53-59.

Atiyah, Michael F., and Ian G. Macdonald. 1969. Introduction to commutative algebra. Reading, MA: Addison-Wesley.

Banaschewski, Bernhard. 1983. "The power of the ultrafilter theorem." Journal of the London Mathematical Society 27 (2): 193-202.

- 1994. "A new proof that 'Krull implies Zorn'." Mathematical Logic Quarterly 40:478-480.

Banaschewski, B., and J. J. C. Vermeulen. 1996. "Polynomials and radical ideals." Journal of Pure and Applied Algebra 113 (3): 219-227.

Berger, Ulrich. 2004. "A computational interpretation of open induction." In Proceedings of the nineteenth annual IEEE symposium on logic in computer science, edited by F. Titsworth, pages 326-334. IEEE Computer Society.

Bishop, Errett. 1967. Foundations of constructive analysis. New York: McGrawHill.

Bishop, Errett, and Douglas Bridges. 1985. Constructive analysis. Springer.

Blechschmidt, Ingo. 2017. Using the internal language of toposes in algebraic geometry. Doctoral dissertation, University of Augsburg.

Boileau, André, and André Joyal. 1981. "La logique des topos." Journal of Symbolic Logic 46 (1): 6-16.

Brewer, Jim, and Fred Richman. 2006. "Subrings of zero-dimensional rings." In Multiplicative ideal theory in commutative algebra: A tribute to the work of Robert Gilmer, edited by James W. Brewer, Sarah Glaz, William J. Heinzer, and Bruce M. Olberding, pages 73-88. New York: Springer Science+Business Media.

Campbell, Paul J. 1978. "The origin of 'Zorn's Lemma'." Historia Mathematica 5:77-89.

Cederquist, Jan, and Thierry Coquand. 2000. "Entailment relations and distributive lattices." In Logic Colloquium '98: Proceedings of the annual European summer meeting of the Association for Symbolic Logic, Prague, Czech Republic, August 9-15, 1998, edited by Samuel R. Buss, Petr Hájek, and Pavel Pudlák, volume 13 of Lecture notes in logic, pages 127-139. Natick, MA: A. K. Peters.

Coquand, Thierry. 1997. "A note on the open induction principle." Technical report, University of Gothenburg. URL: www. cse. chalmers. se/ coqua nd/open.ps.

- 2009. "Space of valuations." Annals of Pure and Applied Logic 157:97-109. 
Coquand, Thierry, Lionel Ducos, Henri Lombardi, and Claude Quitté. 2009. "Constructive Krull dimension. I: Integral extensions." Journal of Algebra and Its Applications 8 (1): 129-138.

Coquand, Thierry, and Henri Lombardi. 2002. "Hidden constructions in abstract algebra: Krull dimension of distributive lattices and commutative rings." In Commutative ring theory and applications, edited by M. Fontana, S.-E. Kabbaj, and S. Wiegand, volume 231 of Lecture notes in pure and applied mathematics, pages 477-499. Reading, MA: Addison-Wesley.

- 2006. "A logical approach to abstract algebra." Mathematical Structures in Computer Science 16:885-900.

Coquand, Thierry, Henri Lombardi, and Stefan Neuwirth. 2019. "Latticeordered groups generated by an ordered group and regular systems of ideals." Rocky Mountain Journal of Mathematics 49 (5): 1449-1489.

Coquand, Thierry, Henri Lombardi, and Marie-Françoise Roy. 2005. "An elementary characterisation of Krull dimension." In From sets and types to topology and analysis, edited by L. Crosilla and P. Schuster, volume 48 of Oxford logic guides, pages 239-244. Oxford University Press.

Coquand, Thierry, and Stefan Neuwirth. 2017. "An introduction to Lorenzen's 'Algebraic and logistic investigations on free lattices' (1951)." Preprint. URL: https://arxiv. org/abs/1711.06139.

Coquand, Thierry, and Henrik Persson. 1998a. "Gröbner bases in type theory." In Types for proofs and programs, edited by T. Altenkirch, B. Reus, and W. Naraschewski, pages 33-46. Springer.

_. 1998b. "Integrated development of algebra in type theory." Calculemus and Types Workshop, 98.

Coquand, Thierry, Giovanni Sambin, Jan Smith, and Silvio Valentini. 2003. "Inductively generated formal topologies." Annals of Pure and Applied Logic 124:71-106.

Coste, Michel, Henri Lombardi, and Marie-Françoise Roy. 2001. "Dynamical method in algebra: Effective Nullstellensätze." Annals of Pure and Applied Logic 111 (3): 203-256.

Crosilla, Laura, and Peter Schuster. 2014. "Finite methods in mathematical practice." In Formalism and Beyond: On the Nature of Mathematical Discourse, edited by G. Link, volume 23 of Logos, pages 351-410. Boston, MA, and Berlin: Walter de Gruyter.

Curi, Giovanni. 2010. "On some peculiar aspects of the constructive theory of point-free spaces." Mathematical Logic Quarterly 56 (4): 375-387.

Eisenbud, David. 1995. Commutative algebra with a view toward algebraic geometry, volume 150 of Graduate texts in mathematics. New York: Springer.

Español, Luis. 1982. "Constructive Krull dimension of lattices." Revista de la Academia de Ciencias Exactas ... Zaragoza (2) 37:5-9.

Fine, Arthur. 1993. "Fictionalism." Midwest Studies in Philosophy 18:1-18. Fourman, Michael, and Robin Grayson. 1982. "Formal spaces." In The L. E. J. Brouwer Centenary Symposium (Noordwijkerhout, 1981), volume 110 
of Studies in logic and the foundations of mathematics, pages 107-122. Amsterdam: North-Holland.

Goldman, Oscar. 1951. "Hilbert rings and the Hilbert Nullstellensatz." Mathematische Zeitschrift 54 (2): 136-140.

Hertz, Paul. 1922. "Über Axiomensysteme für beliebige Satzsysteme. I. Teil: Sätze ersten Grades." Mathematische Annalen 87 (3): 246-269.

—. 1923. "Über Axiomensysteme für beliebige Satzsysteme. II. Teil: Sätze höheren Grades." Mathematische Annalen 89 (1): 76-102.

_. 1929. "Über Axiomensysteme für beliebige Satzsysteme." Mathematische Annalen 101 (1): 457-514.

Hodges, Wilfrid. 1979. "Krull implies Zorn." Journal of the London Mathematical Society 19:285-287.

Howard, Paul, and Jean Rubin. 1998. Consequences of the Axiom of Choice. Providence, RI: American Mathematical Society.

Jacobsson, Carl, and Clas Löfwall. 1991. "Standard bases for general coefficient rings and a new constructive proof of Hilbert's basis theorem." Journal of Symbolic Computation 12 (3): 337-372.

Johnstone, Peter T. 1982. Stone spaces (Cambridge studies in advanced mathematics 3). Cambridge University Press.

- 2002. Sketches of an elephant: A topos theory compendium, volume 2, volume 44 of Oxford logic guides. Oxford: Clarendon Press.

Kemper, Gregor, and Thsen Yengui. 2019. "Valuative dimension and monomial orders." URL: https://arxiv.org/abs/1906.12067

Krull, Wolfgang. 1929. "Idealtheorie in Ringen ohne Endlichkeitsbedingung." Mathematische Annalen 101 (1): 729-744.

Kunz, Ernst. 1991. Algebra. Braunschweig: Vieweg.

Lal, Harbans. 1971. "A remark on rings with primary ideals as maximal ideals." Mathematica Scandinavica 29:72.

Legris, Javier. 2012. "Paul Hertz and the origins of structural reasoning." In Universal logic: An anthology: From Paul Hertz to Dov Gabbay, edited by Jean-Yves Béziau, Studies in universal logic, pages 3-10. Basel: Birkhäuser.

Lombardi, Henri. 2002. "Dimension de Krull, Nullstellensätze et évaluation dynamique." Mathematische Zeitschrift 242:23-46.

Lombardi, Henri, and Claude Quitté. 2015. Commutative algebra: Constructive methods: Finite projective modules, volume 20 of Algebra and applications. Dordrecht: Springer Netherlands.

Lorenzen, Paul. 1950. "Über halbgeordnete Gruppen." Mathematische Zeitschrift 52 (1): 483-526.

_. 1951. "Algebraische und logistische Untersuchungen über freie Verbände." Journal of Symbolic Logic 16 (2): 81-106.

_. 1952. "Teilbarkeitstheorie in Bereichen." Mathematische Zeitschrift 55 (3): $269-275$.

-1953. "Die Erweiterung halbgeordneter Gruppen zu Verbandsgruppen." Mathematische Zeitschrift 58 (1): 15-24. 
. 1953. "Eine Bemerkung über die Abzählbarkeitsvoraussetzung in der Algebra." Mathematische Zeitschrift 57:241-243.

. 2017. "Algebraic and logistic investigations on free lattices." Transl. by Stefan Neuwirth of Lorenzen 1951. URL: https://arxiv. org/abs/ 1710.08138

Mines, Ray, Fred Richman, and Wim Ruitenburg. 1988. A course in constructive algebra. Universitext. New York: Springer.

Moore, Gregory H. 1982. Zermelo's axiom of choice: Its origins, development, $\mathcal{E}$ influence. Mineola, NY: Dover Publications 2013. Unabridged republication of the work originally published as volume 8 in the series "Studies in the history of mathematics and physical sciences" by Springer-Verlag, New York.

Negri, Sara. 2014. "Proof analysis beyond geometric theories: from rule systems to systems of rules." Journal of Logic and Computation 26 (2): 513-537.

Negri, Sara, and Jan von Plato. 1998. "Cut elimination in the presence of axioms." Bulletin of Symbolic Logic 4 (4): 418-435.

Perdry, Hervé. 2004. "Strongly Noetherian rings and constructive ideal theory." Journal of Symbolic Computation 37 (4): 511-535.

Perdry, Hervé, and Peter Schuster. 2011. "Noetherian orders." Mathematical Structures in Computer Science 21:111-124.

- 2014. "Constructing Gröbner bases for Noetherian rings." Mathematical Structures in Computer Science 24:e240206.

Raoult, Jean-Claude. 1988. "Proving open properties by induction." Information Processing Letters 29 (1): 19-23.

Rathjen, Michael. 2005. "Generalized inductive definitions in constructive set theory." In From sets and types to topology and analysis: Towards practicable foundations for constructive mathematics, edited by Laura Crosilla and Peter Schuster, volume 48 of Oxford logic guides, pages 23-40. Oxford: Clarendon Press.

Richman, Fred. 1974. "Constructive aspects of Noetherian rings." Proceedings of the American Mathematical Society 44:436-441.

Rinaldi, Davide. 2014. Formal methods in the theories of rings and domains. Doctoral dissertation, University of Munich.

Rinaldi, Davide, and Peter Schuster. 2016. "A universal Krull-Lindenbaum theorem." Journal of Pure and Applied Algebra 220:3207-3232.

Rinaldi, Davide, Peter Schuster, and Daniel Wessel. 2017. "Eliminating disjunctions by disjunction elimination." Bulletin of Symbolic Logic 23 (2): 181-200.

. 2018. "Eliminating disjunctions by disjunction elimination." Indagationes Mathematicae (N.S.) 29 (1): 226-259.

Rinaldi, Davide, and Daniel Wessel. 2018. "Extension by conservation. Sikorski's theorem." Logical Methods in Computer Science 14 (4:8): 1-17.

. 2019. "Cut elimination for entailment relations." Archive for Mathematical Logic 58 (5-6): 605-625. 
Sambin, Giovanni. 2003. "Some points in formal topology." Theoretical Computer Science 305 (1-3): 347-408.

Satyanarayana, Motupalli. 1967. "Rings with primary ideals as maximal ideals." Mathematica Scandinavica 20:52-54.

Scholz, Heinrich. 1919. "Die Religionsphilosophie des Als-ob." Annalen der Philosophie I (1): 27-113.

Schuster, Peter. 2012. "Induction in algebra: A first case study." In 2012 27 th annual ACM/IEEE symposium on logic in computer science, pages 581-585. IEEE Computer Society Publications. Proceedings, LICS 2012, Dubrovnik, Croatia.

—. 2013. "Induction in algebra: A first case study." Logical Methods in Computer Science 9 (3): 20.

Scott, Dana. 1954. "Prime ideal theorems for rings, lattices, and Boolean algebras." Bulletin of the American Mathematical Society 6o (4): 390.

- 1971. "On engendering an illusion of understanding." Journal of Philosophy 68:787-807.

- 1974. "Completeness and axiomatizability in many-valued logic." In Proceedings of the Tarski Symposium (Proc. Sympos. Pure Math., Vol. xxv, Univ. California, Berkeley, Calif., 1971), edited by Leon Henkin, John Addison, C. C. Chang, William Craig, Dana Scott, and Robert Vaught, pages 411-435. Providence, RI: American Mathematical Society.

- 1973. "Background to formalization." In Truth, syntax and modality (Proc. Conf. Alternative Semantics, Temple Univ., Philadelphia, Pa., 1970), volume 68 of Studies in logic and the foundations of mathematics, edited by Hugues Leblanc, pages 244-273. Amsterdam: North-Holland. Seidenberg, Abraham. 1974. "What is Noetherian?" Rendiconti del Seminario Matematico e Fisico di Milano 44:55-61.

Simpson, Stephen G. 2009. Subsystems of second order arithmetic, second edition. Perspectives in Logic. Cambridge: Cambridge University Press.

Steinitz, Ernst. 1910. "Algebraische Theorie der Körper." Journal für die reine und angewandte Mathematik 137:167-309.

Vaihinger, Hans. 1922. Die Philosophie des Als Ob: System der theoretischen, praktischen und religiösen Fiktionen der Menschheit auf Grund eines idealistischen Positivismus. 7. u. 8. Aufl. Leipzig: Verlag von Felix Meiner.

- 1924. The philosophy of 'as if': A system of the theoretical, practical and religious fictions of mankind. Translated by C. K. Ogden. London: Routledge \& Kegan Paul.

Wessel, Daniel. 2018. "Points, ideals, and geometric sequents." Technical report, University of Verona.

. 2020. "A note on connected reduced rings." Journal of Commutative Algebra. Forthcoming. URL: https://projecteuclid.org/euclid.j $\mathrm{ca} / 1561363253$

Yengui, Ihsen. 2008. "Making the use of maximal ideals constructive." Theoretical Computer Science 392:174-178.

- 2015. Constructive commutative algebra: Projective modules over poly- 
nomial rings and dynamical Gröbner bases, volume 2138 of Lecture notes in mathematics. Cham: Springer.

Zermelo, Ernst. 1904. "Beweis, daß jede Menge wohlgeordnet werden kann." Mathematische Annalen 59:514-516.

-. 1908. "Neuer Beweis für die Möglichkeit einer Wohlordnung." Mathematische Annalen 65:107-128.

Zorn, Max. 1935. "A remark on method in transfinite algebra." Bulletin of the American Mathematical Society 41:667-670.

Open Access This chapter is licensed under the terms of the Creative Commons Attribution 4.0 International License (http://creativecommons.org/licenses/by/4.0/), which permits use, sharing, adaptation, distribution and reproduction in any medium or format, as long as you give appropriate credit to the original author(s) and the source, provide a link to the Creative Commons license and indicate if changes were made.

The images or other third party material in this chapter are included in the chapter's Creative Commons license, unless indicated otherwise in a credit line to the material. If material is not included in the chapter's Creative Commons license and your intended use is not permitted by statutory regulation or exceeds the permitted use, you will need to obtain permission directly from the copyright holder. 